\title{
Avaliação de carcaça de bovinos da raça Tabapuã com ultrassonografia
}

\author{
Rodrigo Zaiden Taveira ${ }^{1,2}$; Otávio Cordeiro de Almeida ${ }^{2}$; Osvaldo José da Silveira \\ Neto'; Alliny das Graças Amaral ${ }^{1}$; Daniel Bueno Dias ${ }^{3}$; Joyce Silva de Barros ${ }^{4}$; \\ Guilherme Brunno de Medeiros Leal ${ }^{5 *}$
}

\author{
${ }^{1}$ Professor Doutor, Faculdade de Zootecnia. Universidade Estadual de Goiás. Brasil. E-mail: \\ rodrigozaiden@gmail.com; oswaldo_neto@hotmail.com; allinyamaral@gmail.com \\ ${ }^{2}$ Professor Doutor, Escola de Ciências Agrárias e Biológicas. Pontifícia Universidade Católica. Goiás. Brasil. \\ E-mail: otávio@pucgoias.edu.br \\ ${ }^{3}$ Zootecnista, Escola de Ciências Agrárias e Biológicas. Pontifícia Universidade Católica (PUC). Goiás. Brasil. \\ E-mail: danielbuenodias@hotmail.com \\ ${ }^{4}$ Zootecnista e Médica Veterinária, Escola de Ciências Agrárias e Biológicas. Pontifícia Universidade Católica. \\ Goiás.Brasil.E-mail: joycebarros@veterinaria.med.br \\ ${ }^{5 *}$ Mestrando em Zootecnia, Departamento de Zootecnia. Universidade Federal de Goiás. Goiás. Brasil. \\ *Autor para correspondência, E-mail: gmzootecnia@gmail.com
}

\begin{abstract}
RESUMO. As medidas de ultrassom possibilitam o conhecimento do nível de musculosidade, da espessura de gordura subcutânea do animal e do grau de marmorização da carne através da mensuração no animal vivo. O objetivo deste trabalho foi avaliar características de carcaça de 20 animais da raça Tabapuã, quantificando os valores médios e desvios-padrões das características de carcaça e estimar correlações simples entre o peso corporal e as medidas ultrassonográfica. As características de carcaça medidas por ultrassom foram área de olho de lombo (AOL), espessura de gordura subcutânea (EGS), mensuradas entre a região da $12^{\circ}$ e $13^{\circ}$ costelas, marmoreio (MAR), relação: altura x largura do contrafilé (RATIO), AOL/100, e EGS/100. As médias estimadas para ao peso corporal, AOL, AOL/100, RATIO, MAR, EGS e EGS/100 foram: $472,23 \pm 140,80 ; 75,78 \pm 21,17 ; 16,33 \pm 2,63 ; 0,45 \pm 0,04 ; 1,03 \pm 0,19 ; 2,33 \pm 0,77 ; 0,52$ $\pm 0,11$, respectivamente. $\mathrm{O}$ valor da AOL encontrada indica animais com alto rendimento de cortes cárneos. As correlações significativas encontradas foram de 0,81 entre a AOL e o peso corporal, 0,70 entre o RATIO e o peso corporal, 0,77 entre o RATIO e AOL, 0,58 entre a EGS e o peso, 0,78 entre EGS e AOL, 0,53 entre EGS e RATIO, 0,41 entre MAR e EGS e 0,70 entre EGS e EGS/100. As correlações significativas foram existentes provavelmente em função dos animais possuírem capacidade suficiente para permitir maior peso e deposição de gordura.
\end{abstract}

Palavras chave: Precocidade, maciez da carne, rendimento

\section{Carcass evaluation of Tabapuã breed bovines using ultrasound}

\begin{abstract}
Measures ultrasound enable the knowledge level of muscularity, the thickness of subcutaneous fat of the animal and the degree of marbling of the meat by measuring in vivo animal. The objective of this study was to evaluate carcass traits of 20 animals Tabapuã, quantifying the mean values and standard deviations of carcass traits and estimate simple correlations between body weight and ultrasound measurements. Carcass characteristics were measured by ultrasound ribeye area (AOL), fat thickness (EGS), obtained between the 12th and 13th rib, marbling score (MAR), ratio: height $\mathrm{x}$ width of the counterfilet (RATIO), AOL/100 and EGS/100. The estimates for body weight, AOL, AOL/100, RATIO, MAR, EGS and EGS/100 averages: $472.23 \pm 140.80$, $75.78 \pm 21.17,16.33 \pm 2.63,045 \pm 0.04,1.03 \pm 0.19,2.33 \pm 0.77,0.52 \pm 0.11$, respectively. The value of AOL found indicates animals with high yield of meat cuts.
\end{abstract}


Significant correlations were 0.81 between AOL and body weight, between 0.70 RATIO and body weight, between 0.77 RATIO and AOL, 0.58 between EGS and weight, between 0.78 and EGS AOL, 0.53 RATIO between EGS and 0.41 between SEA and SFT and 0.70 between EGS and EGS/100. Significant correlations were probably due to the existing animals possessing sufficient to allow greater weight and fat deposition capacity.

Key words: Precocity, meat tenderness, yield

\section{Introdução}

Os processos de melhoramento genético vêm crescendo desde a introdução das raças zebuínas no Brasil, por meio das importações. No princípio, utilizavam somente as características raciais, pois alguns criadores julgavam ser de grande importância essas características (ABCZ, 2013). Novas técnicas dos processos de melhoramento genético foram sendo desenvolvidas com a inovação tecnológica, e os zebuínos aumentando a participação nestes.

Como o peso por si só não determina adequadamente o valor de um animal produtor de carne, existe busca por tecnologias e mensurações que indiquem com maior precisão a composição da carcaça. Nesse contexto a ultrassonografia para avaliação de carcaça consolidou-se como técnica viável, não invasiva, não destrutiva, acurada e de custo aceitável para esta função. E que já é utilizada com sucesso em programas de melhoramento genético (Yokoo et al., 2009).

As medidas de ultrassom possibilitam o conhecimento do nível de musculosidade, da espessura de gordura subcutânea do animal e do grau de marmorização da carne pela mensuração no animal vivo da área de olho de lombo (AOL), espessura de gordura subcutânea (EGS) e marmoreio (MAR), RATIO (relação: altura $x$ largura do contrafilé), respectivamente (Valero et al., 2014).

As raças exploradas ou o manejo adotado influenciam o peso final de abate, a precocidade e o acabamento do animal (Rotta et al., 2009). Algumas características importantes da carcaça, como os cortes cárneos, a cobertura de gordura, os subprodutos da desossa, a maciez, entre outras, estão intimamente relacionadas à idade de abate do animal, ao seu grau de acabamento e ao seu peso de carcaça.

O objetivo desta pesquisa foi avaliar características de carcaça de 20 animais da raça Tabapuã, quantificando os valores médios e desvios padrões das características de carcaça, sendo: AOL; AOL/100; RATIO; Marmoreio;
EGS; EGS/100, desses novilhos; e estimar correlações simples entre o peso corporal e as medidas ultrassonográfica entre estas.

\section{Material e Métodos}

O trabalho foi desenvolvido com animais da raça Tabapuã, pertencentes ao plantel da Fazenda Rancho Tabapuã, localizada no município de Hidrolândia - Goiás. Foi utilizado um delineamento inteiramente casualizado, com 20 novilhos com idade média de 23 meses. Os animais foram selecionados aleatoriamente, sem observação de aspectos genealógicos e de desempenho prévio.

Os animais se encontravam em regime extensivo de criação, sendo que as principais gramíneas utilizadas para o pastejo dos animais eram constituídas basicamente de Brachiaria brizantha cv. Marandu e Panicum maximum cv. Mombaça, suplementados com mistura mineral por meio de cochos distribuídos pelo piquete.

O aparelho de ultrassom utilizado para realizar a coleta das imagens foi um Aloka SSD500 VET, com sonda linear de $17 \mathrm{~cm}$, de 3,5 $\mathrm{MHz}$ e um acoplador acústico com um sistema de captura de imagens. O software utilizado foi o Bia International Feedlot e as imagens foram coletadas por técnica certificada pela UGC (Ultrasound Guidelines Council).

Realizou-se as mensurações de ultrassonografia em tempo real, coletando as imagens de ultrassom da AOL e da EGS, mensuradas entre a região da $12^{\circ}$ e $13^{\circ}$ costelas, transversalmente ao músculo Longissimus dorsi, MAR e RATIO.

Para a coleta das imagens da AOL e EGS, foi utilizado óleo vegetal e um acoplante ("stand off"), para garantir o contato acústico entre a sonda linear e o corpo do animal.

Durante o período de março de 2013 a outubro de 2013, foi observado dos animais, realizando-se as pesagens, controlando-se o regime alimentar, efetuando o manejo sanitário e o manejo reprodutivo. 
As análises estatísticas foram realizadas mediante a utilização do programa estatístico computacional R (Versão 2.15.2). As correlações simples foram estimadas por meio da correlação de Pearson, utilizando-se nível de significância de $5 \%$.

\section{Resultados e Discussão}

A tabela 1 apresenta os valores médios, desvios-padrões e coeficientes de variação referentes ao peso corporal, AOL, AOL/100, RATIO, marmoreio, EGS e EGS/100.

O peso médio encontrado foi de $472,2 \mathrm{~kg} \pm$ $140,8 \mathrm{~kg}$. Pode ser percebida bastante variação no comportamento dessa variável, com coeficiente de variação estimado em $29,8 \%$, evidenciando muitas influências não controláveis na estimação do peso corporal, entre elas diferentes tipos de pastagens e suplementação as quais esses animais tiveram acesso antes da avaliação, do nascimento até ao desmame.

Tabela 1. Análise descritiva referente as mensurações de carcaça de 20 novilhos da raça Tabapuã pertencentes ao rebanho Rancho do Tabapuã, localizado no município de Hidrolândia, Goiás.

\begin{tabular}{lcc}
\hline & Média \pm DP & CV $(\%)$ \\
\hline Peso $(\mathrm{Kg})$ & $472,23 \pm 140,80$ & 29,81 \\
AOL $\left(\mathrm{cm}^{2}\right)$ & $75,78 \pm 21,17$ & 27,93 \\
AOL/100 & $16,33 \pm 2,63$ & 16,10 \\
RATIO & $0,45 \pm 0,04$ & 8,88 \\
Marmoreio & $1,03 \pm 0,19$ & 18,44 \\
EGS & $2,33 \pm 0,77$ & 33,04 \\
EGS/100 & $0,52 \pm 0,11$ & 21,15 \\
\hline
\end{tabular}

A AOL apresentou valor médio de 75,8 $+21,2$ $\mathrm{cm}^{2}$. Esse valor encontra-se inferior e superior aos $116,4 \mathrm{~cm}^{2}+69,8 \mathrm{~cm}^{2}$ ao observado por Suguisawa et al. (2006) e Suguisawa \& Soares, (2006) trabalhando com animais da raça Caracu em regime de semi confinamento e Meirelles et al. (2013) trabalhando com animais da raça Tabapuã em regime de confinamento. Essa diferença entre os trabalhos ocorre possivelmente devido aos agrupamentos genéticos e sistema de manejo nutricional divergentes. A estimação desse parâmetro é de extrema importância já que possui correlação positiva com os demais cortes cárneos de interesse econômico da carcaça, além de ser uma medida facilmente obtida por meio da ultrassonografia. Segundo Suguisawa (2002), acima de $75 \mathrm{~cm}^{2}$ indica animais de alto rendimento de cortes cárneos.

Tendo em vista o RATIO, o valor médio foi de 0,45 e coeficiente de variação de $8,9 \%$, constituindo-se na menor porcentagem de todas as características avaliadas nesse estudo. Se tratando de uma característica investigativa se os animais serão produtores de carne.

O marmoreio médio encontrado foi de 1,0. O valor médio do marmoreio apresenta-se menor e maior que os 2,37 $\pm 0,67$ e 0,99 registrados por Menezes (2013) com animais da raça nelore em regime alimentar extensivo e Marques et al. (2006) com animais confinados provenientes do cruzamento entre Nelore $\mathrm{x}$ Red Angus. A avaliação do marmoreio das carcaças é de extrema importância já que influencia diversos aspectos que agregam qualidade na carcaça.

A EGS registrada foi de 2,3 $\mathrm{mm}$. Esse valor médio da EGS encontra-se menor e maior que os $4,8 \pm 1,26$ e 1,9 $\pm 1,36$ registrados por Zorzetto et al. (2013) com animais da raça Nelore em regime de confinamento e Yokoo et al. (2009) também em animais da raça Nelore e em regime intensivo. A maioria dos frigoríficos brasileiros trabalham com mínimo de $3 \mathrm{~mm}$ tentando evitar que ocorra o encurtamento dos sarcômeros das fibras, o que resultaria numa carne mais dura e com menor valor no mercado.

A tabela 2 apresenta os valores das correlações simples entre as características avaliadas.

As correlações significativas $(\mathrm{P}<0,05)$ encontradas nesse estudo foram de 0,81 entre a AOL e o peso corporal, 0,70 entre o RATIO e o peso corporal, 0,77 entre o RATIO e a AOL, 0,58 entre a EGS e o peso, 0,78 entre EGS e AOL, 0,53 entre EGS e RATIO, 0,41 entre marmoreio e EGS e 0,70 entre EGS e EGS/100. 
Tabela 2. Correlações simples entre as características avaliadas provenientes de novilhos da raça Tabapuã pertencentes ao rebanho Rancho do Tabapuã, localizado no município de Hidrolândia, Goiás.

\begin{tabular}{lccccccc}
\hline & Peso & AOL & AOL/100 & RATIO & Marmoreio & EGS & EGS/100 \\
\hline Peso & - & & & & & & \\
AOL & $0,81^{*}$ & - & & & & & \\
AOL/100 & $-0,38$ & 0,19 & - & & & & \\
RATIO & $0,70^{*}$ & $0,77^{*}$ & 0,04 & - & & & \\
Marmoreio & 0,21 & 0,32 & 0,05 & 0,08 & - & - & \\
EGS & $0,58^{*}$ & $0,78^{*}$ & 0,12 & $0,53^{*}$ & $0,41^{*}$ & - \\
EGS/100 & $-0,001$ & 0,35 & 0,39 & 0,10 & 0,40 & $0,70^{*}$ & - \\
\hline
\end{tabular}

*Correlação significativa $(\mathrm{P}<0,05)$.

A correlação simples de 0,81 encontrada entre a AOL e o peso corporal evidencia existir associação forte e positiva entre essas duas características, já que os animais mais pesados foram possuidores de maiores valores de AOL.

A estimativa da correlação simples de 0,70 entre o RATIO e o peso corporal, caracteriza-se por ser positiva e de alta magnitude, sugerindo que maiores valores de RATIO associaram-se com maiores pesos.

Entre o RATIO e a AOL foi encontrada estimativa de correlação simples de 0,77 , também se caracterizando por ser positiva e de alta magnitude, indicando ter havido associação entre maiores valores de RATIO e AOL.

A correlação simples entre a EGS e o peso corporal foi de 0,58 , sendo também considerada forte e de alta magnitude, evidenciando que os animais mais pesados foram possuidores de maiores valores para EGS, provavelmente em função de serem os animais mais velhos e já possuírem maturidade suficiente para permitir maior deposição dessa gordura.

Em estudo realizado por Silva et al. (2003) avaliando as relações entre medidas de ultrassonografia na carcaça de 22 bovinos da raça Nelore, obtiveram correlação positiva, de 0,87 para as medidas de AOL e EGS. Resultados semelhantes foram obtidos neste trabalho, em que encontrou correlação simples de 0,78 entre a AOL e a EGS também positiva e de alta magnitude. Indica que os animais possuidores de maiores valores para AOL também apresentaram maiores estimativas para a EGS, possuindo carcaça mais valorizada no mercado.

A correlação entre a EGS e o RATIO foi de 0,53 , também alta magnitude e positiva. Trata-se de uma característica investigativa onde os animais mais acabados também são maiores produtores de carne.
Já a correlação entre a EGS e o MAR foi de 0,41 , condiz possivelmente de animais que já estão bem acabados e são produtores de carne mais macia e mais valorizada no mercado.

\section{Conclusão}

A AOL média encontrada foi divergente devido aos grupamentos genéticos apresentados e o regime alimentar em que os animais se encontravam. Os valores obtidos estão acima do que indicam animais com alto rendimento de cortes cárneos. Onde os animais da pesquisa podem produzir melhor rendimento no frigorífico.

O RATIO mensurado prediz a animais que possam entrar em confinamento, proporcionando ótimo ganho em peso e rendimentos de cortes cárneos.

Para EGS o valor foi inferior ao mínimo exigido pelo frigorífico, penalizando a carcaça com essa característica, que resultaria numa carcaça dura, com pouco acabamento e um menor valor comercial. A medida de MAR da pesquisa condiz com a carcaça com um grau de maciez mediano. Onde ele condiz com agregar qualidade na carcaça.

As correlações fenotípicas positivas encontradas foram AOL e peso, RATIO e peso, EGS e peso, RATIO e AOL, EGS e AOL, EGS e RATIO, MAR e EGS e EGS e EGS/100, são correlações altas, que associam animais mais pesados a animais bem acabados, com maiores rendimentos cárneos e possuidores de carcaça mais valorizada.

\section{Referências Bibliográficas}

ABCZ. (2013). Programa de Melhoramento Genético de Zebuínos. Controle de Desenvolvimento Ponderal. Associação Brasileira de Criadores de Zebu, Uberaba. 
Marques, J. A., Prado, I. N., Moletta, J. L., Prado, I. M., Prado, J. M., Macedo, L. M. A., Souza, N. E. \& Makoto, M. (2006). Características físico-químicas da carcaça e da carne de novilhas submetidas ao anestro cirúrgico ou mecânico terminadas em confinamento. Revista Brasileira de Zootecnia, 35, 15141522.

Meirelles, S.L. C., Vieira, G. R., Estopa, A. C.,Baena, M. M., Furtado, T. C., Gonçalves, T.M. (2013). Desempenho e características de carcaça de bovinos da raça Tabapuã terminados em confinamento. Anais... X Simpósio Brasileiro de Melhoramento Animal. Uberaba, Minas Gerais.

Menezes, G. R. O., Nieto, L. M., Rosa, A. N., Nobre, P.R. C., Silva, L.O. C.,Gondo, A. (2013). Tendências genéticas para características de carcaça ao sobreano na raça Nelore - Programa Geneplus - Embrapa. Anais... X Simpósio Brasileiro de Melhoramento Animal. Uberaba, Minas Gerais.

Rotta, P. P., Prado, R. M., Prado, I. N., Valero, M. V., Visentainer, J. V. \& Silva, R. R. (2009). The effects of genetic groups, nutrition, finishing systems and gender of Brazilian cattle on carcass characteristics and beef composition and appearance: a review. Asian-Australasian Journal of Animal Sciences, 22, 1718-1734.

Silva, S. L., Leme, P. R., Pereira, A. S. C. \& Putrino, S. M. (2003). Correlações entre características de carcaça avaliadas por ultrasom e pós-abate em novilhos Nelore, alimentados com altas proporções de concentrado. Revista Brasileira de Zootecnia, $32,1236-1242$.

Suguisawa, L. (2002). Ultra-sonografia para predição das características e composição da carcaça de bovinos. Universidade de São Paulo.
Suguisawa, L., Mattos, W. R. S., Oiliveira, H. N., Silveira, A. C., Arrigoni, M. B. \& Souza, A. A. (2006). Correlações simples entre as medidas de ultra-som ea composição da carcaça de bovinos jovens. Revista Brasileira de Zootecnia, 35, 169-176.

Suguisawa, L. \& Soares, W. R. M. (2006). Ultrasonografia para predição da composição da carcaça de bovinos jovens. Revista Brasileira de Zootecnia, 35, 177-185.

Valero, M. V., Prado, R. M., Zawadski, F., Eiras, C. E., Madrona, G. S. \& Prado, I. N. (2014). Propolis and essential oils additives in the diets improved animal performance and feed efficiency of bulls finished in feedlot. Acta Scientiarum. Animal Sciences, 36, 419-426.

Yokoo, M. J. I., Werneck, J. N., Pereira, M. C., Albuquerque, L., Koury Filho, W., Sainz, R. D., Lobo, R. B. \& Araújo, F. (2009). Correlações genéticas entre escores visuais e características de carcaça medidas por ultrassom em bovinos de corte. Pesquisa Agropecuária Brasileira, 44, 197-202.

Zorzetto, R. S., Silveira, J. S., Lima, M. C.,Cucco, D. C., Correa, L. B., Silva, S.L. E, Zanetti, M.A. (2009). Caracterização do desenvolvimento de parâmetros de carcaça avaliados por ultrassonografia em bovinos Nelore confinados. Anais...17 $7^{\circ}$ Simpósio Internacional de Iniciação Científica. Universidade de São Paulo. Pirassununga São Paulo.

\section{Recebido em Setembro 7 de 2015}

Aceito em Outubro 19, 2015

License information: This is an open-access article distributed under the terms of the Creative Commons Attribution License, which permits unrestricted use, distribution, and reproduction in any medium, provided the original work is properly cited. 